\title{
ARTICOLI
}

\section{La scrittura di Cettina Natoli Ajossa tra passioni tardoromantiche e presentimenti decadenti}

\section{The Writings of Cettina Natoli Ajossa Among late-Romantic Passions, and Decadent Premonitions}

\author{
Daniela Bombara \\ Università di Messina \\ daniela.bombara63@gmail.com
}

\begin{abstract}
The aim of this article is to bring to the attention of the scholars the writings of Cettina Natoli Ajossa (1867-1913), Sicilian noblewoman, who published several novels in which the Romantic paradigm is questioned and the crisis of the Nineteenth-Century world is highlighted. Particularly relevant the autobiographical memoir of Messina earthquake: it focuses upon the bitter conflict between classes, giving us the meaning of the end of an era.
\end{abstract}

Keywords: Romanticism in crisis, Cettina Natoli Ajossa, Sicilian women writers, Messina earthquake

L'esistenza di questa nobildonna siciliana non sembra riservare particolari sorprese: nata a Messina nel $1867^{1}$, mostra una precoce vocazione di scrittrice, e viene quindi mandata a studiare a Firenze, ma torna in Sicilia al compimento della maggiore età per sposare il marchese Giovanni Ajossa di Palmi. Il marito non le impedisce di coltivare i suoi interessi: sarà pittrice, musicista, socia dell'Accademia

\footnotetext{
${ }^{1}$ La data di nascita non è presente nelle poche biografie esistenti, ma si deduce dalle parole di Giovanni Canevazzi: "Il suo primo romanzo Margherita Royn [...] rimonta a qualche anno fa, quando la marchesa non era ancora ventenne, ed era solo da pochi mesi divenuta sposa" (124).
} 
Peloritana di Messina, giornalista e autrice di diversi romanzi, storie d'amore tormentate, che non firma mai col suo nome; i contemporanei ricordano soprattutto Margherita Royn. Una storia d'amore, pubblicato nel 1886 come Eola, Fiori di serra (1890) e Fiocco di neve (1896) come Espero.

Un quadro equilibrato, nel quale esigenze personali e familiari si integrano senza quegli slittamenti, o aperte ribellioni, che possiamo riscontrare nelle vicende biografiche di altre intellettuali dell'epoca: la formazione culturale di Natoli, limitata agli anni dell'adolescenza, è diretta genericamente alle "arti", fra cui la scrittura²; e si tratta comunque, almeno ad uno sguardo superficiale, di racconti sentimentali, o di prosa pedagogica, come nel moraleggiante Serate d'inverno del 1901, indirizzato alle figlie.

Si consideri però, in primo luogo, l'imponenza di una produzione narrativa che annovera, compresi i tre già citati, ben sei romanzi editi - Finch'io viva e più in là (1899) Granelli inutili (1902), Verso la vita (1912); gli inediti Nadia, Sacrifizio, Foglie di rosa; infine Le mie cinque giornate di Messina 28-12-1908/1-1-1909 (1914), intenso racconto autobiografico delle vicende seguite al terremoto che devasta la città siciliana. Rilevante inoltre la collaborazione con periodici quali "La Vita italiana", diretta da Angelo De Gubernatis, e "Flegrea", ideata da Riccardo Forster per agganciare l'ambiente napoletano alla cultura europea. L'autrice non è quindi ignota: agli esordi "Andrea Maffei, la definiva una scrittrice piccola di età, ma grande di sentimenti [...]. Ruffini, De Gubernatis e molti altri [...] la spronarono a proseguire" (Canevazzi, 1896, 122). Segue un quasi assoluto silenzio degli studiosi, e la figura di Cettina Natoli trova posto unicamente in repertori: Bandini Buti $(1941,22)$ riprende con qualche inesattezza il profilo del già citato Canevazzi; nel volume Donne a Messina troviamo una breve voce redatta da Giovanni Molonia (2014, 154-155). Costituiscono una rilevante eccezione i lavori di Anna Santoro, che nel 1987 antologizza, nel suo Narratrici italiane dell'Ottocento, alcune pagine dell'autrice, proponendo un primo orientamento critico:

\footnotetext{
${ }^{2}$ Nella Dedica alla principessa di San Teodoro, che precede Fiocco di neve, Natoli dichiara che il suo libro ha "deficienze, ineguaglianze, povertà di stile, [ma] quando si vive lontani da qualsiasi luce d'intelletto, privi di emozioni e di godimenti spirituali, in una progrediente atrofizzazione d'ogni energia - trovare in sè la forza di creare un qualunque mondo ideale e cercare di dare ad esso una forma artistica, credilo, mia cara, è pure qualcosa non spregevole" (7-8) La professione d'ignoranza è un topos nella scrittura femminile siciliana fra ' 800 e ' 900 (Bombara, 2017, 82, 86), excusatio non petita per giustificare l'incursione nella Repubblica delle Lettere. Gli stessi pseudonimi - non solo Espero ed Eola ma, come c'informa Canevazzi $(1896,124)$ anche Dolores, Marchesa nera, Cetty - sminuiscono l'importanza di un'attività letteraria che bisogna in qualche modo occultare. Ma c'è forse anche il senso di una distanza incolmabile, e ancora più avvertita dopo l'Unità, fra isola e Continente, il secondo luogo di un più intenso e produttivo scambio culturale e di maggiori opportunità editoriali (Sui modelli educativi riservati alle donne nell’Ottocento vedi Soldani 1989).
} 
L'interesse dei romanzi della Natoli sta nella svolta, cosciente e dichiarata, dal romanzo d'azione al romanzo psicologico di gusto decadente per la descrizione dei personaggi e degli ambienti. In più c'è la ricerca dell'evento strano, dell'accadimento inaspettato, non più appartenente alla tipologia del romanzo popolare o d'azione, ma teso verso l'inchiesta nel malato, nel torbido (79).

In un saggio successivo sulla narrativa a firma femminile di fine '800 Santoro afferma che Natoli focalizza l'antagonismo fra i sessi, motivo topico dell'epoca, "soprattutto fissando $\mathrm{i}$ ruoli assegnati dalla società e vissuti dai protagonisti come ineluttabili" (1992, 115). Significativo quindi lo sguardo critico della marchesa, scrittrice "apparentemente convenzionale" - è sempre Santoro a parlare - ma che in realtà "rompe canoni e oper[a] svolte significative per la narrativa del tempo". Il suo "svelamento della società "bene" si compie utilizzando i modi espressivi della "morbosità decadente" $(2006,761)$; Natoli descrive quindi un mondo in disfacimento, in cui "i protagonisti, uomini e donne, perdono gli aloni misteriosi, e per alcuni eccitanti, che possedevano presso altri e più famosi scrittori (si pensi a D'Annunzio) per rivelarsi maschere meschine di un'epoca e di un ambiente in piena crisi"' (761). Da un lato quindi i romanzi dell'autrice esprimono l'impossibilità della passione sincera in un contesto sociale aristocratico dove predominano interesse e culto dell'apparenza; ma Natoli va oltre il paradigma romantico per denunciare l'inanità dei suoi stessi protagonisti, appassionati ma nevrotici, incapaci di opporsi realmente alle regole di una società distopica che intimamente disprezzano, ma soprattutto impossibilitati a vivere l'esistenza senza mediazioni, che siano le costruzioni letterarie o l'attitudine razionalizzante.

Ne è un esempio la vicenda di Margherita Royn (1886), che ama Riccardo Olivieri, artista povero; ma il legame va contro gli obblighi di casta e gli interessi economici della famiglia, per cui i due innamorati finiscono per sposare ognuno persone diverse. La ragazza si strugge nel ricordo, e sarà uccisa dal marito geloso che, in una macabra scena, la divora di baci poiché ha pronunciato, in un delirio provocato dalla febbre, il nome dell'amato. In realtà la protagonista è bloccata da un'ipersensibilità che le impedisce di affrontare la realtà, esperita solo attraverso il filtro di letture appassionate:

Il suo temperamento era delicato e febbrile: aveva bisogno di emozioni forti, violente, che la lasciassero affranta. [...] Era una natura debole, languida, straordinariamente sensibile, eccessivamente nervosa, che si alterava, si esaltava, si trasformava per la tensione dei nervi, per la sovraeccitazione della fantasia. In quel momento Margherita stava col braccio appoggiato sulla scrivania, pensando; era nell'abbandono che succedeva alle più forti esaltazioni del cuore. [...] La manina bianca ricadeva, stanca, sopra un libro aperto, e l'unghia rosea dell'indice era ancora ferma là, dove aveva sottolineate quelle parole: che cosa è la vita senza l'amore?! (7-9) ${ }^{3}$.

\footnotetext{
${ }^{3}$ L'estrema, morbosa sensibilità di Margherita è raccontata con compiacimento dannunziano: "S'impressionava, si alterava per uno scricchiolio della sedia, per qualche cosa che strisciava sul tavolo,
} 
La stessa relazione fra Margherita e Riccardo è vissuta quasi esclusivamente tramite una corrispondenza epistolare convulsa, che finisce per alterare la realtà: la protagonista, per ottenere dall'amato conferme, ne aizza la gelosia inscenando per lettera la strenua opposizione paterna e la minacciosa presenza di possibili pretendenti, costruendo quindi un doppio romanticizzato del reale, e identificando se stessa con l'eroina perseguitata ${ }^{4}$. Quando effettivamente il problema si presenterà, e sarà comunicato per iscritto, Riccardo non riesce più a crederci, e si ritrae disgustato di fronte ad una commistione fra vita ed elaborazione fantastica per lui incomprensibile. La protagonista, cresciuta all'ombra del pessimismo materno di matrice leopardiana (ancora un modello letterario), si nutre per reazione di melodrammi verdiani, romanze di Tosti, Luigi Denza, Fabio Campana. La sua è un'esistenza strozzata, virtuale, nella quale l'ascolto, gli spettacoli teatrali, la lettura di romanzi e lettere, avidamente rilette sino a perdere ogni funzione comunicativa - "rileggeva ogni giorno le lettere di lui, gli scriveva lungamente, aprendo intiero l'animo suo, sfogando la piena dei suoi affetti; ma dopo stracciava quelle lettere e ne disperdeva tutti i pezzettini volanti" (105) - sostituiscono la vita reale, che reclama però i suoi diritti nella persona del marito, Florestano, amante possessivo ed intransigente ${ }^{5}$. In realtà anche questo personaggio è estremo, nel suo folle desiderio di ottenere affetto ad ogni costo da una donna che gli sfugge, ed anche il suo comportamento esprime

per lo stridio d'una finestra che si chiudeva; dopo poco, sbatacchiava, ella stessa, un uscio, rumorosamente; $[\ldots]$ stringeva forte i denti per farne uscire un suono stridulo, strisciante, e provava un'acre sensazione di piacere, una soddisfazione arcana, ma forte" (9), ma la fonte primaria è senz'altro il personaggio di Roderick Usher, in The Fall of the House of Usher (1839) di Edgard Allan Poe, autore diffuso in Italia tramite Baudelaire, ma anche gli italiani Camerini, Guerrazzi, Maineri (Melani, 2006). Margherita condivide con il proprietario della casa Usher l'attaccamento ossessivo alla casa, con cui entra in simbiosi: nello specifico si tratta del suo studiolo, che fa riprodurre identico ad ogni trasferimento; lo spazio domestico è intriso di memorie care ma al tempo stesso fagocitanti, al punto da impedire l'azione della giovane donna, confinata al passato. Sulla lettura come attività pericolosa, nell'ottica del tempo, poiché crea un eccesso di sensibilità, vedi Gusso (1999, 64-74).

${ }^{4}$ Per il motivo della 'letteraturizzazione' del reale in Natoli ed altre scrittrici siciliane fra '800 e ' 900 vedi Bombara (2017, 66-91).

${ }^{5}$ Il tema del libro/feticcio, che sostituisce la passione amorosa e al tempo stesso induce a relazioni ex lege, è centrale in Madame Bovary di Flaubert (1856), romanzo che costituisce certamente la fonte primaria per molte vicende di adulterio nelle opere del secondo Ottocento; Emma, come Margherita, si circonda di immagini letterarie per "far fronte ad una sofferenza che è sempre la stessa sotto aspetti diversi: il tormento della vicinanza" (Tanner, 1990, 298), quindi l'impossibilità di accettare il contatto con un marito radicalmente 'altro', anche a livello puramente fisico. Il libro è in effetti, in questa e in altre narrazioni, "un surrogato mediale dell'amore che resta non comunicabile e non dicibile" (Scherpe, $2015,144)$, per quanto strumento comunicativo imperfetto e generatore di gravi fraintendimenti (Scherpe si riferisce nello specifico al Werther e alle Affinità elettive di Goethe): "La lettera e il libro come media destinati a condizionare lo sviluppo dell'intreccio recano fin dall'inizio i segni dell'esito tragico - il medium trasmette e collega ma separa anche e può perfino irresistibilmente dividere" (Scherpe, 2015, 146). 
un disequilibrio fra reale e ideale. Le scene coniugali di passione violenta, al limite dello stupro, costituiscono un leit motiv del romanzo:

[S]e la strinse così forte che ne sentì i battiti del cuore, e posò lungamente le sue labbra febbricitanti sulle labbra di lei, tumide, semiaperte, rosse, che parevano sanguinanti (119-120).

Mi prese un desiderio febbrile di stringerti fino a soffocarti, di vederti morire almeno per opera mia (135).

E pensava con gioia infinita di stringersela forte al cuore e baciarla sulla fronte, sugli occhi, sulla bocca, succhiandole le labbra lungamente, voluttuosamente. [...] D'un tratto Margherita si sentì soffocare, le mancò il respiro, voleva gridare, ma Florestano le aveva chiusa la bocca coi suoi baci lunghi, violenti, l'aveva presa fra le sue braccia, come una bambina, se la stringeva al cuore e nell'impeto della passione febbrile le diceva mille cose incoerenti, sconnesse, ma piene di passione colme di ebbrezza, traboccanti di voluttà (165).

La tensione fra Eros e Thanatos esplode nella cannibalica scena finale; la devastazione omicida di Florestano ricorda certamente il delitto di Bouvard, che nell'omonimo racconto di Tarchetti profana il cadavere della donna inutilmente amata:

[C]on un grido di desiderio infinito, sollevatala fra le sue braccia si mise a baciarla, a baciarla sulla fronte, sugli occhi; nel collo, nelle labbra, ardentemente, furiosamente, disperatamente, rudemente [...]; la stringeva ancora più violentemente, con passione brutale, con rabbia disperata. Ella si dibatteva debolmente, le mancava il respiro, avea gli occhi sbarrati, e sotto quella stretta violenta la debole personcina si stirava, si contorceva convulsa. [...] L'esile corpo della vittima si contrasse nervosamente ancora, poi si distese, raffreddandosi, irrigidendosi; gli occhi vitrei, spalancati, erano iniettati di sangue. [...] In quel triste silenzio si udiva lo schioccare dei baci furenti di Florestano. Era una scena voluttuosa e feroce, d'amore e d'odio, di baci e di morte (230-231).

Margherita, consunta dalla passione, incapace di reggere il confronto con la realtà, nella sua magrezza esasperata - "Gli occhi neri si erano ingranditi, dilatati nella magrezza del viso lungo e sfinito; il naso s'era fatto più sottile, la pelle pareva stirata sulle ossa, la bocca s'era allargata sulle guancie sbiadite" (219); "gli occhi neri e profondi, dallo sguardo infinitamente triste, s'erano incavati in un cerchio nerissimo" (225) - è vicina alla Fosca tarchettiana; in entrambi i casi "la malattia, soprattutto psichica, si accampa con forte ambiguità superando la concezione di un 'negativo' naturalista, per coinvolgere insieme livello artistico e componenti inconsce" (Crotti, 1992, 44). L'inettitudine e la follia dei personaggi è indizio dello svuotamento di senso di una classe alto-borghese e aristocratica che, concluso lo slancio ideale del Risorgimento, ha smarrito un quadro di riferimento valoriale (Billiani, 2008, 480-499), per quanto non sia presente in Natoli alcun riferimento concreto alla questione; ma nei lineamenti distrutti di Margherita, martirizzata dalla passione "i contorni delicati del volto erano indecisi, sfumati: pareva cominciassero a disfarsi" 
(207) - appare almeno in tutta evidenza il crollo delle idealità romantiche, inadeguate ormai a rappresentare una realtà in cui una cieca volontà di far del male emerge dall'interiorità degli individui; violenza che dopo pochi anni si diffonderà all'intero corpo sociale ${ }^{6}$.

Meno interessante il macchinoso Fiori di serra del 1890, il cui contenuto si esprime tutto nel titolo: narra infatti di una coppia dai "delicati sentimenti", che "si trovavano sempre in urto con le volgarità della vita comune" (9), personaggi che "si creano un mondo indefinibile che nessuno comprende, nemmeno essi stessi" (59); esseri delicati e gentili, che forse l'autrice tratta con lieve ironia. Guido ama con trepidazione e angoscia Emma, per quel "vizio della sua mente che era l'analisi di tutto, l'analisi che, andando sempre in traccia della verità, uccide la fede, il sentimento, le illusioni" (11), e ne è riamato allo stesso modo; i due sperimentano uno scacco vitale di matrice scapigliata - si pensi all'Arnoldo D. de Una scommessa di Gualdo, incapace di creare per una "tabe interiore" (Desideri, 1989, 972) che lo costringe ad esaminare razionalmente ogni spunto creativo, ogni moto del cuore vicino anche al predecadentismo europeo (Huysmans): amano quindi l'idea dell'amore sublime più che l'amore in sé. Ma l'estetismo conduce alla follia: indotti a sospettare tradimenti, in un frenetico avvicendarsi di balli in maschera con scambi di ruolo, tentati duelli e possibili suicidi, i due finiscono per separarsi, ed Emma impazzirà per il dolore.

Fiocco di neve (1896) è un'opera certamente più matura, che mette in campo nuove tematiche: il rifiuto del ruolo sottomesso della donna, e l'urgenza insopprimibile del desiderio fisico femminile, tacitato da una società che cerca di imbrigliarlo nel legame matrimoniale, o lo disprezza nelle relazioni extraconiugali. Amelia sopporta con gelida indifferenza una famiglia anaffettiva; evita quindi l'amore silenzioso del cugino Enrico e la passione di Alberto, giovane affascinante ma senza mezzi, per sposare un 'buon partito', il principe Valdemaro, che le possa garantire la fuga dal contesto familiare. Ma la "schiacciante freddezza" (67) del marito, per il quale "la donna è la macchina destinata alla soddisfazione delle sue follie [...], una cosa sciocca sempre" (74), le provoca un acuto rimpianto dell'amore, soffocato sul

\footnotetext{
${ }^{6}$ È in ogni caso l'adulterio, da cui originano malattia e disgregazione della psiche, a porsi in primo luogo come elemento di disgregazione del corpo sociale; esso introduce infatti una commistione e 'adulterazione' di ruoli che mette in discussione l'istituto della famiglia borghese, basata sul matrimonio come 'contratto' per "congiungere armoniosamente modelli di passione e modelli di proprietà" (Tanner, 1990, 27). La consunzione di Margherita, come "i processi di rarefazione, disintegrazione e perdita di contorno subiti da Emma" (Tanner, 1990, 316), o ancora la follia autodistruttiva di Anna Karenina di Tolstoj, evidenziano lo smarrimento identitario della donna adultera, priva di uno status riconosciuto, e destinata ad una nullificazione che agisce come critica implicita ad un sistema valoriale diventato ormai puro involucro di leggi e precetti, "sempre più svuotato di senso [...]; lo squallore del nulla penetra sin nelle strutture più consuete del matrimonio e dell'ordine borghese" (Luperini, 2015, 227).
} 
nascere, per il sensibile Alberto, sposato a sua volta con Elda, vanitosa e incapace di comprenderlo ${ }^{7}$. Le due coppie si incontrano, determinando un gioco di attrazioni incrociate vagamente somigliante alle Affinità elettive (1809) goethiane. Amelia non cede mai interamente agli assalti di un desiderio che ritorna però costantemente, poiché è proprio l'attrazione che suscita in Alberto a rafforzare la sua identità di donna reificata dall'indifferenza e la brutalità del marito, a riscattarla dal sentirsi "una povera cosa destinata a passare nel mondo inosservata ed inutile" (158):

[...] nel mio cuore s'agita una sorda ribellione, divampa un grande incendio... Anch'io voglio vivere (111).

[S]entiva ancora repressa ma non estinta la fiamma che le aveva acceso le fibre; e. infatti, spesso sorgeva da un fondo oscuro del suo essere, come da un segreto vulcano, un soffio di desiderii ardenti, un fremito di voluttà ignorate (147-8).

[Sentiva] sempre la repressione di tutta sè stessa non compresa, non amata mai (168).

[L]e strette di mano furtive eran vere scosse elettriche, seguite da brividi e fremiti, che si propagavano lungo i loro corpi (189).

Il desiderio di Alberto la pungeva (202).

Elda invece cede a Valdemaro, con il quale avrà una bimba, Bianca, che anni dopo si innamora del figlio di Amalia. Al motivo, riconoscibilmente dannunziano, dell'incesto, già adombrato dal rapporto affettuoso fra la protagonista e il cugino Enrico, non è dato particolare rilievo; sembra che l'autrice voglia sottolineare, più che le complicanze psicologiche a cui dà luogo in D'Annunzio il "sororalismo" amoroso (Goudet, 1976, 254), l'ideale, sia pure espresso in negativo, del legame amoroso come relazione fra pari. In questo senso sono da intendersi le ultime parole del romanzo, quando Amelia risponde con "impetuosa fierezza" al figlio le chiede se, dopo anni, ami ancora Alberto: "Lo amo perchè è il solo uomo che mi abbia compresa, il solo che mi abbia apprezzata, il solo che mi abbia veramente amata" (320).

\footnotetext{
${ }^{7}$ Amelia definisce Valdemaro "un mutilato del cuore, e crede che tutti siano così; oltre ad essere brutale, è incosciente. Non sa, non comprende, non intuisce nulla di quel che si passa dentro di me; mi crede una cosa, non una persona" (110). È possibile che il tema dell'adulterio come risarcimento amoroso sia mutuato da Anna Karenina (1877), diffuso ben presto in Italia grazie alla traduzione di Domenico Ciampoli del 1887 per Treves; se è vero però che Karenin non può concepire "l'idea che lei [Anna] abbia pensieri, desideri diversi dai suoi, insomma una sua vita personale che non coincide con la sua" (Malcovati, 2015, 121), il personaggio russo, "di straordinaria statura tragica nella sua solitudine, umiliazione, disperazione" (Malcovati, 2015, 107), ha ben altra complessità e statura morale rispetto a Valdemaro. Quello di Amelia è inoltre un adulterio non consumato, potremmo dire un "adulterio non adultero", prendendo in prestito il titolo di un capitolo (24-26) dal libro di Emilia Fiandra, Desiderio e tradimento (2005); si consideri però che il tradimento, per quanto virtuale, individua la legittimità del desiderio femminile ed evidenzia la personalità della donna, oppressa dal vincolo matrimoniale; anche in questo caso, dunque, l'atto appare trasgressivo ed "a soccombere è il tessuto sociale che si sgretola intorno agli adulteri” (Fiandra, 2005, 32).
} 
E arriviamo al 1908, quando Cettina Natoli, come tanti, perde un familiare, la figlia ventenne Alfrida, nel terremoto di Messina. Ma lei racconta l'evento, firmando col suo vero nome, in una prosa asciutta che descrive 'per sottrazione' l'orrore del disastro, come assenza delle cose, prima che delle persone:

Un urlo - che non mi parve neppure venuto da lui [dal marito] - con le disperate parole "non c'è più nulla!" rintronò nelle mura crollate e si spense fuori nel gran vuoto. Egli, dove ancora avrebbe dovuto trovare due stanze, sentì il vuoto nell'aria fredda che lo colpì: e vide le stelle! (6).

Al dolore della perdita si aggiunge una vera e propria discesa agli inferi: la perdita della casa e dei beni comporta una sempre maggiore degradazione della nobile coppia, costretta a dividere spazi ristretti nella folla ostile, che rinfaccia loro il privilegio aristocratico. Una danza macabra, intrisa di freddo e di terrore, di malignità e dolore, dove il disastro appare democratico come la morte livellatrice. I "signori prepotenti" (26), rifugiati con gli altri in un ferryboat, sono sbeffeggiati, depredati, gli viene impedito di bere e mangiare:

Tutta quella gente ci era ostile, ci guardava sogghignando, aveva la soddisfazione della nostra sventura; per quell'odio di classe, che giunge fino ad impietrire il cuore nelle più gravi sciagure, essa assisteva al nostro pianto col sorriso beffardo sulle labbra. "Ora siamo tutti gli stessi” andavano ripetendo, guardandoci in faccia come una sfida (38).

Cettina Natoli muore nel 1913, non fa in tempo a registrare i rivolgimenti provocati dalla Grande Guerra, ma ha già descritto con lucidità un nodo problematico del secolo appena iniziato - il disgregarsi del privilegio aristocratico di fronte al malessere e alla volontà di protagonismo delle classi basse - in un libriccino che vuole esporre, come recita l'esergo, "Nient'altro che la verità, semplicemente raccontata" (3). 


\section{BIBLIOGRAFIA}

Bandini Buti, M. (1941-1942). Aiossa Natoli Concettina. In M. Bandini Buti, Poetesse e scrittrici. Enciclopedia bio-bibliografica (vol. II, p. 22). Roma: Istituto Editoriale Italiano C. Tosi.

Billiani, F. (2008). Delusional Identities: The Politics of the Italian Gothic and Fantastic in Iginio Ugo Tarchetti's Trilogy Amore nell'arte and Luigi Gualdo's Short Stories, "Allucinazione", "La canzone di Weber" and "Narcisa". Forum for Modern Language Studies, 44, 4, 480-499.

Bombara, D. (2017). Leggere per vivere. La lettura come paradigma interpretativo del reale nelle scrittici siciliane dell'Otto-Novecento. In A. Nemesio (Ed.), Il lettore nel testo (pp. 66-91). Torino: Trauben.

Canevazzi, G. (1898). Cettina Natoli-Ajossa (Espero). In G. Canevazzi, Profili di scrittrici italiane (pp. 119-133). Lecce: Lazzaretti.

Crotti, I. (1992). Lo sperimentalismo di Tarchetti. In I. Crotti, R. Ricorda (Eds.), Scapigliatura e dintorni (pp. 37-47). Padova: Piccin.

Desideri, G. (1989). Il fantastico. In A. Asor Rosa (Ed.), Letteratura italiana. Storia e geografia (vol. III: L'età contemporanea, pp. 969-998). Torino: Einaudi.

Fiandra, E. (2005). Desiderio e tradimento. L'adulterio nella narrativa dell'Ottocento europeo. Roma: Carocci.

Goudet, J. (1976). D’Annunzio romanziere. Firenze: Olschki.

Gusso, V. (1999). La lettura corporea. Il rapporto tra fisicità e pratica del leggere in una selezione di brani di autori ottocenteschi. Biblioteche oggi, 4, 64-74.

Luperini, R. (2015). Un cambiamento di paradigma: trasformazione della figura dell'adultera e fine del romanzo d'adulterio tra Otto e Novecento. In E. Villari (Ed.), L'adulterio nel romanzo (pp. 221-236). Pisa: Pacini.

Malcovati, F. (2015). Perché non spegnere la candela, quando non c'è più niente da guardare? L'adulterio in Anna Karenina. In E. Villari (Ed.), L'adulterio nel romanzo (pp. 105-136). Pisa: Pacini.

Melani, C. (2006). Effetto Poe. Influssi dello scrittore americano sulla letteratura italiana. Firenze: University Press.

Molonia, G. (2014). Cettina Natoli Ajossa. In M. D’Angelo, G. Molonia (Eds.), Donne a Messina. Storia delle donne come storia della città (pp. 154-155). Messina: MD Edizioni.

Natoli, C. [Eola] (1886). Margherita Royn. Una storia d'amore. Napoli: Tipografia privata.

Natoli, C. [Espero] (1890). Fiori di serra. Palmi: Lopresti.

Natoli, C. [Espero] (1896). Fiocco di neve. Milano: Galli.

Natoli, C. [Espero] (1899). Fin ch'io viva e più in là. Napoli: Pierro e Veraldi.

Natoli, C. [Espero] (1901). Serate d'inverno. Napoli: Pierro e Veraldi.

Natoli, C. [Espero] (1902). Granelli inutili. Rocca S. Casciano: Cappelli.

Natoli, C. [Espero] (1912). Verso la vita. Napoli: Trani.

Natoli, C. (1914). Le mie cinque giornate. Messina 28 dicembre 1908 - 1 gennaio 1909. Napoli: Cimmaruta.

Santoro, A. (1987). Narratrici italiane dell'Ottocento. Napoli: Federico \& Ardia.

Santoro, A. (1992). Narrativa di fine Ottocento: le scrittrici e il pubblico. Italiana, IV, 103-126.

Santoro, A. (2006). Cettina Natoli Ayossa Grifeo. In M. Fiume (Ed.), Siciliane. Dizionario biografico (pp. 761-762). Siracusa: E. Romeo.

Scherpe, K.R. (2015). La lettera "A" e altri media nel romanzo d'adulterio. In E. Villari (Ed.), L'adulterio nel romanzo (pp. 137-156). Pisa: Pacini. 
Soldani, S. (Ed.). (1989). L'educazione delle donne: scuole e modelli di vita femminile nell'Italia dell'Ottocento. Milano: Franco Angeli.

Tanner, T. (1990). L'adulterio nel romanzo. Contratto e trasgressione, trad. G. Pomata (ed. or. Adultery in the Novel. Contract and Transgression, The Johns Hopkins University Press, 1979). Genova: Marietti. 